\title{
6 month prospective audit of Parenteral Nutrition in a general hospital
}

\author{
Carolyn A. Speirs \\ Royal Bolton Hospital NHS Foundation Trust, Minerva Road, Bolton, BL4 OJR, UK
}

A prospective audit of in-patients on Parenteral Nutrition (PN) was carried out over a 6 month period from August 2009, to compare the Trust's PN practices with aspects of the NICE guideline on Nutrition Support for Adults ${ }^{(1)}$ and identify practices or procedures for improvement. Royal Bolton Hospital's procedures for out of hours PN had been changed to improve safety since PN was previously audited.

Dietitians identified patients for audit from their PN referrals. The audit was designed to identify the consultants responsible for care, the wards involved, whether the clinical indications for PN were appropriate ${ }^{(1)}$, risks of re-feeding syndrome, processes (who recommended starting or stopping PN, the day of the week and time it commenced, expected duration of PN, nutritional assessment, infusion regimens) and duration of $\mathrm{PN}$.

The sample size was 34, mainly surgical and critical care patients. PN was recommended by the consultant in 26 cases and jointly by consultant and dietitian in 8 . All patients had appropriate clinical indication(s) for PN, and 32 had their nutritional requirements assessed by the dietitian within 1 working day, the remaining 2 having died before the dietitian could assess them.

PN was commenced mainly on weekdays; 7 commenced during a weekend. Data on time of commencement was not collated but PN was known occasionally to commence in the early hours (05.10am, 01.10am, 06.30am). Patients on PN were located across a number of wards, a few on which medical and nursing skills in PN might have been limited.

Of the sample, 25 commenced PN on a reduced rate (either according to recommendations for prevention and management of refeeding syndrome, or to meet $50 \%$ of energy requirements initially as per NICE) and 4 on full volume. 3 of the 4 patients starting PN at full volume were commenced out of hours before being assessed by the dietitian. 15 patients were predicted to be at risk of re-feeding syndrome.

30 patients had an expected duration of PN of 14 days or less, compared with 20 actual. Access for PN was central line in 25 cases and peripheral in 3. The relative few on peripheral feeding is explained in part by a central line already being in situ. Of the 14 patients on PN for more than 14 days, the regimen was adjusted to cyclical feeding in only 5 cases.

Decision to stop PN was made by the consultant in 15 cases, dietitian in 7 (with agreement of doctors), and joint decision (dietitian and consultant/team) in 5 .

The main findings of this audit compare very well with the NCEPOD report ${ }^{(2)}$ which has since been published, with respect to PN being prescribed appropriately and patients having their nutritional requirements assessed by appropriately trained health professionals (dietitians).

Recommendations made from the audit findings included use only of starter PN regimens at weekends, timings of PN bag changes to be sensitive to patients' needs, limitation of PN to wards with staff skilled in PN, involvement of dietitians in decisions to stop PN, investigation into establishing a dedicated PICC line service, and training in PN for junior doctors.

Since this audit PN is no longer commenced at full volume, more peripheral PN is being carried out, cyclical PN is considered for patients on PN for longer than 2 weeks, PN is taught at a quarterly nutrition study day for nurses and a handbook has been produced for junior doctors.

1. National Institute for Clinical Excellence (2006) Nutrition Support for Adults: Oral Nutrition Support, Enteral Tube Feeding and Parenteral Nutrition. 2. National Confidential Enquiry into Patient Outcome and Death (2010) A Mixed Bag. 